Recherches en didactique des langues et des cultures

Les cahiers de l'Acedle

17-2 | 2020

Recherches collaboratives en didactique des langues

\title{
Le profil et les représentations des étudiants gambiens pour le cours de français langue étrangère
}

Ndèye Maty Paye

\section{OpenEdition}

Journals

Édition électronique

URL : https://journals.openedition.org/rdlc/7802

DOI : $10.4000 /$ rdlc.7802

ISSN : 1958-5772

Éditeur

ACEDLE

Référence électronique

Ndèye Maty Paye, "Le profil et les représentations des étudiants gambiens pour le cours de français langue étrangère ", Recherches en didactique des langues et des cultures [En ligne], 17-2 | 2020, mis en ligne le 27 avril 2020, consulté le 17 octobre 2022. URL : http://journals.openedition.org/rdlc/7802 ; DOI : https://doi.org/10.4000/rdlc.7802

Ce document a été généré automatiquement le 17 octobre 2022.

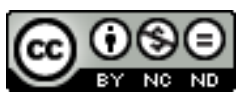

Creative Commons - Attribution - Pas d'Utilisation Commerciale - Pas de Modification 4.0 International - CC BY-NC-ND 4.0

https://creativecommons.org/licenses/by-nc-nd/4.0/ 


\section{Le profil et les représentations des étudiants gambiens pour le cours de français langue étrangère}

Ndèye Maty Paye

\section{Introduction}

1 Pour choisir une langue à diffuser, à promouvoir ou encore à enseigner, quelle qu'elle soit, un ensemble de conditions s'impose : déterminer la situation sociolinguistique, comprendre les politiques linguistiques et éducatives, les habitudes culturelles, la géographie, l'histoire et les représentations de la langue. La sociolinguistique démontre ainsi l'importance du contexte dans tout enseignement/apprentissage, dans toute élaboration de projet didactique, mais aussi dans toute politique linguistique (décision et action). De ce fait, en nous basant sur le français langue étrangère en contexte universitaire gambien, nous tenterons de répondre dans cette contribution aux questions suivantes: dans quel contexte est enseigné le français à l'université gambienne sous l'appellation de FRE 101/ FRE 102? Qui sont les apprenants de FRE 101/102? Quelles sont leurs représentations de la langue et leurs difficultés d'apprentissage. Notre travail s'appuiera sur une enquête par questionnaire réalisée en juin 2017.

\section{La politique linguistique de l'université de Gambie}

2 La politique linguistique permet une gestion des langues, de la prise de décisions (politique) à l'application réelle de ces décisions (planification). A la lecture du document sur les politiques linguistiques de The University of the Gambia (UTG), nous constatons que l'administration universitaire donne une plus-value à la maitrise du français, en considérant l'espace ouest-africain à majorité francophone. Ainsi, le français est une matière obligatoire (GER : General Education Requirements), en référence à la circulaire 
du lundi 9 février 2015. La finalité de cette décision est d'asseoir des compétences en français pour rendre compétitifs les étudiants au niveau du marché international du travail (relations internationales, diplomatie, sciences politiques, interprétariat, commerce, tourisme) après l'anglais, langue d'instruction à l'université gambienne. Nous retrouvons cette volonté dans le passage suivant :

"In order to ensure UTG students and graduates are regionally and internationally competitive and to better serve The Gambia in international platforms with reasonable French language competencies, a decision was taken by Senate to enhance our students' French language competency skills every semester they are enrolled in the UTG. The Gambia is surrounded by French speaking countries in the sub region and most of the professionals in these countries are at least bilingual and do speak French fluently as their first language and do possess English language competency and in some cases a third language. At its meeting held on Monday $9^{\text {th }}$ February 2015, Senate revisited its earlier decision on the French GER courses and after a thorough and exhaustive deliberation, directed as follows :

A French Language competency graduation requirement for all UTG students regardless of major or degree programme.

To ensure competency, all UTG students MUST be exposed to French Language acquisition every semester they are enrolled at the UTG and must pass each enrolled French language course every semester they are enrolled at the UTG and this will be a graduation requirement for all students"

Senate Resolution on French Courses, the University of the Gambia, circulaire universitaire, bureau des inscriptions (registrar), du lundi 09 février 2015.

3 L'anglais est la langue officielle de la Gambie, de facto et non de jure, principale langue d'instruction et d'administration universitaire. L'anglais est alors une langue véhiculaire, au statut de langue seconde. En première année, les étudiants gambiens, apprennent le français sous l'appellation de Elementary French : FRE 101 pour le premier semestre et le FRE 102 pour le deuxième semestre. Au-delà de la première année, ils doivent opter pour un français professionnel, puis pour un français de spécialisation en fonction de leur domaine. Cependant, sept mois après la décision du gouvernement de rendre obligatoire l'apprentissage du français en première année et à la suite de protestations contre ces mesures, l'administration universitaire est revenue sur sa décision en allégeant l'offre linguistique pour le français, en référence à la circulaire du 14 septembre 2015. En effet, certains membres de l'association des étudiants considèrent cette politique comme une action inconstitutionnelle, selon les propos recueillis, le 13 novembre 2015, sur le site All Africa, du secrétaire général de l'association des étudiants UTGSU d'Almamy S. Manga. Le français reste obligatoire (compulsory), pour tous les étudiants. Mais, cette nouvelle orientation s'accompagne de restrictions. En effet, la mesure ne concerne que les matières FRE 101 et FRE 102 (niveau débutant). Tout étudiant, exprimant un intérêt, au-delà de la première année pour le français à un niveau plus avancé, peut le signaler et prolonger ses études durant la période estivale. La faculté de médecine et celle de journalisme dérogent à cette règle par nécessité, puisque les mobilités académiques de leurs étudiants ainsi que leurs spécialisations se font dans la sous-région (ex : le Sénégal francophone).

"At its meeting held on Saturday 12th September, 2015, Senate, deliberated extensively on the French Courses and the decision is, summarized below :

a) That as part of the GER, Foreign Language Requirements, all students offering French must do French 101 and 102 (Compulsory)

c) That those students who demonstrate interest in French beyond the GER level have the option to enroll in higher level French courses during the summer sessions at their own expense and convenience." 
Senate Resolution on French Courses, (Registrar), The University of the Gambia, circulaire universitaire du samedi 14 septembre, 2015, bureau des inscriptions. gambienne, nous avons opté pour un travail de recherche sur le terrain en donnant la parole aux étudiants. L'analyse des représentations sociolinguistiques en contexte didactique est importante, en ce sens. Elles concernent l'idée que les acteurs sociaux se font de leurs pratiques langagières et la signification sociale, qu'ils leur attribuent, selon Blanchet (2007 : 35).

\section{La technique d'enquête}

5 Pour une enquête efficace, nous avons utilisé le cours de Méthodologie de la recherche, selon Alén-Garabato (2011), de l'université Paul Valéry, Montpellier 3. Ainsi, nous avons travaillé avec le logiciel d'enquête Sphinx Plus version 2000. Le logiciel nous a facilité les stades de l'enquête: l'élaboration du questionnaire, la saisie des données, les traitements quantitatifs et qualitatifs à la fois, l'analyse lexicale. Prévue au mois de mars 2016, nous n'avons pu réaliser notre enquête qu'à la fin du mois de juin 2017 à la période des examens du second semestre 2016-2017 au campus universitaire de Brikama en région 2 (Gambie). Nous avons pu rassembler notre corpus en un seul et même jour à savoir le jour de l'examen final des FRE 101 et FRE 102. Soixante étudiants de l'université de Gambie forment notre population d'enquêtés. Nous leur avons soumis un questionnaire composé de 22 questions fermées et ouvertes sur le français langue étrangère en contexte universitaire gambien.Les questions ouvertes nous ont garanti des réponses libres. Les questions fermées nous ont permis de proposer aux enquêtés plusieurs réponses possibles et de choisir librement entre elles pour une information plus standardisée, ce qui nous a permis d'aboutir à la fois à une analyse quantitative, qui éclaire sur les parcours personnels et les perceptions, une analyse quantitative permettant un accès aux statistiques et aux chiffres, une analyse lexicale des items et occurrences les plus citées et leur significativité. Le questionnaire comporte cinq parties principales : la première donne sens aux données socio-biographiques (profils), la deuxième offre un panorama sur l'apprentissage du FRE101/102 à UTG, la troisième est focalisée sur l'(in)sécurité linguistique des apprenants, la quatrième sur les difficultés rencontrées par ces apprenants et la cinquième sur les attentes de nos enquêtés. Nous allons dans ce qui suit, inventorier les résultats obtenus.

\section{Le profil des apprenants de FRE 101 et 102}

6 Notre échantillon est constitué d'une population très jeune dont l'âge ne dépasse pas 30 ans. Nous pouvons le vérifier dans la présentation de certains où la tranche varie plus précisément entre 17 et 28 ans, le plus âgé de notre corpus a effectivement 30 ans. Tandis qu'un seul enquêté $n^{\circ} 56$ n'a pas donné de précision à ce sujet. Contrairement aux années où la population estudiantine était âgée (40 ans et plus), la donne a changé. Nous comprenons par là que le taux de scolarisation a augmenté en Gambie et que la jeunesse est beaucoup plus impliquée dans le sort national en investissant dans les études. Notre population est à majorité de nationalité gambienne $95 \%$ excepté l'enquêté $n^{\circ} 6$ qui est Sierra-léonais. Toutefois, nous avons deux non-réponses. L'élite 
est composée de beaucoup de natifs tandis que la population étrangère estudiantine s'amoindrit. Peut-on envisager un nationalisme progressif de plus en plus intensifié et assumé ? Les personnes interrogées proviennent de diverses filières et sont pour la majorité à la faculté des lettres et des Sciences (School of Arts and Science - SAS), sauf les étudiants des facultés de droit (law school), de médecine, des technologies de l'information (ICT school) et les études infirmières (Nursing). Ils suivent leurs principaux cours en majorité à Brikama, Kanifing, Banjul suivant leurs spécialisations. Ils préparent la licence sous l'appellation anglaise de bachelor degree. Il est vrai que l'université gambienne est très jeune, mais progressivement elle s'autonomise en recrutant moins la ressource humaine étrangère et en misant sur sa propre population. Diverses filières existent et les déplacements vers l'extérieur pour des spécialisations se réduisent du fait d'une offre universitaire gambienne qui s'enrichit au fil des années, grâce à la compétence locale. Il reste à octroyer à celle-ci les moyens d'asseoir leurs compétences par la formation continue et la validation des acquis, sanctionnée des diplômes aux niveaux les plus élevés comme le master et le doctorat. Ce partenariat est en cours de réalisation grâce à la coopération universitaire étrangère, aux universités virtuelles qui dispensent les cours en ligne (plateforme de l'AUF et université virtuelle africaine (AVU)...). La majorité des apprenants à Brikama, pour le FRE 101/102, provient du département de development studies soit $40 \%$, suivi à part égale, soit $10 \%$, des départements de : political Science, médecine (préméd.), english, public health... Notre corpus révèle plus d'étudiants de sexe féminin que masculin, soit $62 \%$ de femmes contre $37 \%$ d'hommes. Les femmes ne sont pas laissées en arrière-plan. Elles sont impliquées dans les débats intellectuels et les processus de décisions.

\section{Les langues étrangères apprises par les étudiants gambiens à UTG}

7 L'anglais est une langue officielle en Gambie, une langue seconde, de scolarisation. C'est une langue d'enseignement, un héritage colonial aussi. Aujourd'hui, il est la langue de l'administration et le véhicule du savoir, au regard de la Constitution Gambienne et de la politique linguistique gambienne. Nous considérons que tous les étudiants le maîtrisent et le possèdent dans leur répertoire plurilingue, Jobe (1993). La première langue étrangère apprise à l'université gambienne à $99 \%$ est le français, suivi de l'arabe. En réalité la position du français est celle d'une première langue étrangère à l'université gambienne, suivi pour une faible part de l'arabe en deuxième position. L'espagnol et le turc n'ont pas été proposés ces semestres passés pour les raisons évoquées cf. supra : la politique linguistique à UTG. Les apprenants valorisent le français car il intègre le programme universitaire. Le FRE 101/102 revêt un caractère obligatoire pour les étudiants en première année de licence. Il s'agit d'une première contrainte, extérieure à la volonté des étudiants. Le français est imposé par l'administration universitaire. Les réponses données montrent que l'administration a pris cette décision dans l'intérêt des étudiants pour des motifs: pratique (large diffusion du français sur la scène internationale avec un large auditoire), pragmatique (économique), culturel (connaissance avec les voisins francophones de l'Afrique de l'ouest), matériel (travail, argent, étude, voyages, conférences, coopérations...), comme le souligne, OCKOVA (2007 : 268-270). Les étudiants, malgré ce forcing, expliquent euxmêmes leur intérêt pour la langue française. Elle est utile, à en croire les réponses, pour 
leur projet professionnel. Elle est importante car la francophonie de proximité est à prendre en compte. De même, nous avons observé pour certains étudiants une vraie passion pour le français.

Illustration : Textes extraits de la question ${ }^{\circ} 6$. Why 1 ?

Observation $n^{\circ} 6$ : it's part of my course requirements.

Observation $n^{\circ} 21$ : my dream is to work in the Gambian embassy in Senegal

besides I love French language.

Observation $n^{\circ} 24: 1$ love to be able to speak it as my first foreign language.

\section{Les représentations linguistiques du français chez les étudiants gambiens}

\section{L'appréciation positive du français}

8 Le goût pour la langue française s'avère réel avec $92 \%$ de francophiles contre $7 \%$. L'image du français reste alors positive dans cette institution anglophone. Nous avons aussi procédé à une analyse lexicale en demandant aux enquêtés d'associer au minimum 5 adjectifs au français. La question était: question $n^{\circ} 10$ : Please choose five adjectives for qualifying French language? L'analyse lexicale montre que le français est jugé intéressant (cité 15 fois) selon les mots des apprenants et insiste surtout sur son statut international, officiel. Il peut aider lors des communications exogènes. Le français a ici une valeur pratique, fonctionnelle. Il est considéré comme une langue véhiculaire, Calvet (1999), de large diffusion, une opportunité, qui peut les aider lors des mobilités académiques. Les adjectifs : helpful et international cités une fois chacun, ont été associés au français. Quelques réponses à cet effet, peuvent être citées :

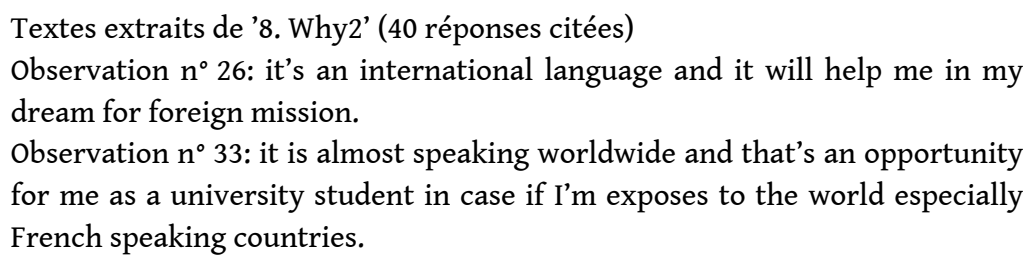

9 Certains étudiants expliquent leur intérêt pour le français en lui associant leur projet professionnel. En ce sens, ils expriment leur volonté d'être bilingue (anglais/français) pour pouvoir travailler dans des organisations internationales comme l'ONU ou la diplomatie gambienne.

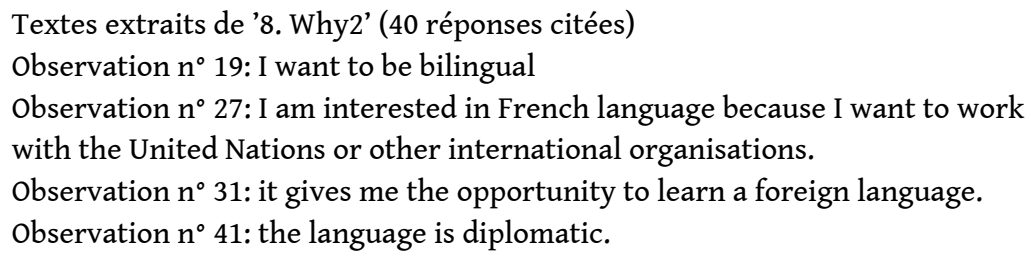

Pour d'autres étudiants, c'est la valeur esthétique de la langue qui est privilégiée. Selon eux, le français est une langue belle, agréable à parler, qui fait qu'ils se sentent bien. L'analyse lexicale montre clairement les adjectifs associés à la langue française pour évoquer sa beauté : beautiful 4 fois, Nice 4 fois, magnifique 2 fois, appealing 2 fois, captivating 1 fois, agreable 1 fois. C'est une langue à laquelle est attribuée une valeur éducative et 
morale, c'est-à-dire qu'elle donne accès à l'instruction, au savoir-vivre et savoir-faire : educative 4 fois, polite 1 fois. Une valeur affective est associée au français qui rappelle les sentiments et les émotions. Les adjectifs associés à ce sujet au français sont les suivants : amazing 1 fois, enjoyable 1 fois, formidable 1 fois, genial 1 fois, romantic, 1 fois, sentimental 1 fois, impressive 1 fois, loveable 1 fois, passionnant 1 fois. Enfin, une valeur génétique est accordée au français qui est jugé comme apparenté à l'anglais ou encore sa facilité ou difficulté est ici de mise. Easily 1 fois, perfect 1 fois, rich 1 fois, soft 1 fois, standard 1 fois.

\section{L'appréciation négative du français}

$117 \%$ des répondants ont exprimé un désintérêt pour le français qu'ils rejettent et au sujet duquel ils éprouvent un sentiment négatif, en insistant sur sa difficulté (valeur génétique). Ils admettent leur incapacité à le parler. Par ailleurs, l'analyse lexicale du corpus offre une vision générale de l'appréhension du français avec des adjectifs négatifs qui lui sont associés : unimportant 1 fois, complicated 2 fois, ardnous 1 fois, brain cracking 1 fois, petty 1 fois, difficult 1 fois, edge 1 fois. En résumé, l'apprentissage du FRE 101/102 s'accompagne d'évaluations majoritairement positives mais aussi négatives du français auquel est accordé des valeurs, génétique, esthétique, morale, pragmatique, fonctionnelle, Calvet (1999: 75). La compréhension des perceptions s'avère ainsi indispensable pour trouver des solutions destinées à améliorer les conditions de l'apprentissage/enseignement du FLE à UTG, améliorer l'offre linguistique et accroître la motivation et des apprenants et des enseignants par ricochet.

\section{Les difficultés rencontrées par les étudiants gambiens dans le cadre de l'apprentissage du FLE}

Les étudiants en majorité (67\%) éprouvent des difficultés dans leur apprentissage, même si $30 \%$ déclarent ne pas en avoir. Une analyse lexicale des items et occurrences les plus cités montre une prédominance de l'aspect oral dans les difficultés citées: parler (speaking, cité 7 fois), lire (reading, cité 4 fois), prononcer (pronounciation cité 9 fois), épeler (cité 3 fois). En revanche, l'écrit a été peu cité (3 fois). Dans la liste des justifications données, nous avons remarqué d'autres obstacles expliqués au-delà de la compétence linguistique. L'environnement linguistique, surtout familial et amical, ne donne pas aux apprenants l'opportunité de pratiquer le français. Le français est limité d'après eux, aux séances de classe. De plus, un problème de matériel pédagogique est souligné. Par exemple, à l'absence de laboratoire de langue à UTG, s'ajoute le manque de tables. Cela engendre un retard considérable sur les cours de 3 heures par semaine. Certains étudiants avouent qu'il leur est souvent impossible de se présenter au cours car ils travaillent simultanément dans d'autres structures. Or, l'absence au cours n'est pas tolérée par les professeurs. D'autres encore déclarent que le temps accordé au français est trop court. 


\section{Les recommandations données par les étudiants gambiens}

13 A la suite des limites citées, nous leur avons donné la possibilité de s'exprimer librement afin de recueillir leurs suggestions. Celles-ci pourraient contribuer à améliorer leurs conditions d'apprentissage. 44 réponses ont été enregistrées et se résument en ces points :

- Pourvoir des salles de classe suffisantes et bien équipées (matériel audiovisuel, tables et chaises...).

- Une plus grande focalisation sur l'enseignement de l'oral

- Sensibiliser les étudiants sur l'intérêt du français

- Encourager la recherche en français

- Redynamiser le club de français

- Organiser des séjours linguistiques vers les pays francophones

\section{L'avenir optimiste du français à UTG}

L'avenir semble prometteur pour l'enseignement/apprentissage du français à l'université de Gambie. A la question 20: do you want to learn French after FRE101/102 at UTG? La réponse est majoritairement positive. Beaucoup d'étudiants souhaitent que des cours à des niveaux plus avancés, 103, 104, 201, 202... soient réintégrés au programme universitaire pour leur permettre d’améliorer leur compétence en langue française. Ils sont $78 \%$ à exprimer cette volonté contre $7 \%$. Il est aussi à noter que $15 \%$ des répondants ne se prononcent pas sur le sujet. La majorité de notre population d'enquête, à savoir 90 \% espère un avenir radieux du français à l'université gambienne, contre $4 \%$ qui expriment une réserve sur la question. Les arguments positifs et optimistes à l'égard du français à l'université sont nombreux. Certains étudiants affirment avoir une connaissance antérieure du français et indiquent que l'étudier à l'université a augmenté leur passion pour la langue. D'autres ne tarissent pas d'éloges sur leurs enseignants qu'ils trouvent actifs, dynamiques, ponctuels, qualifiés. Ensuite, nous avons ceux qui trouvent que le français ne peut-être qu'un atout pour l'université pour la simple et bonne raison que toute université digne de ce nom devrait proposer à ses apprenants au minimum deux langues étrangères. Un autre motif donné est qu'il est difficile d'ignorer la langue française, vu la proximité géographique de la Gambie avec la francophonie. Pour terminer sur les opinions favorables au français, nous avons pu observer que les apprenants manifestent un réel intérêt pour la francophonie, institution qu'ils souhaitent connaître davantage.

\section{Les réserves par rapport au français}

Même s'ils constituent une minorité, des apprenants de FRE 101/102 avec une motivation quasi-nulle pour l'apprentissage du français sont répertoriés dans notre corpus. Nous constatons toujours que $7 \%$ de notre échantillon, soient 42 enquêtés, ne sont pas convaincus. Ils disent ne pas aimer le français, ne croient pas en l'avenir du français à UTG. Ils ne souhaitent pas poursuivre l’apprentissage du français dans les niveaux supérieurs. Selon leurs propos, les étudiants ne prennent pas au sérieux les 
cours de français. Ils pensent que certains ne l'apprennent que pour réussir et avoir de bons résultats avant de le jeter aux oubliettes. La complexité de la langue est citée comme facteur bloquant. L'enquêté 24 pose comme condition que les enseignants soient plus flexibles par rapport aux absences des étudiants, d'alléger les emplois du temps trop souvent chargés et de ne pas organiser des cours de rattrapage le week-end. Ainsi, il est utile de comprendre ce rejet du français pour arriver à des solutions pouvant transformer la francophobie en une francophilie qui fait balayer toute réticence.

\section{Conclusion}

Les résultats obtenus montrent que le français jouit en majorité d'une représentation positive à l'université gambienne. En ce sens, les données de 2010 lors de notre thèse coïncident avec celles de 2017. A aucun moment de cette enquête, le français n'a été qualifié de langue ennemie, colonisatrice ou langue d'ingérence. Aujourd'hui, cet esprit nationaliste, bien que présent, a tendance à s'estomper. Gomez (2013) l'explique en se basant sur la littérature gambienne. Depuis le traité d'amitié entre le Sénégal et la Gambie en 1991, nous observons une relation de partenariat malgré quelques zones de turbulences. Et c'est le français en Gambie qui en sort gagnant. Nous avons eu, en juin 2017, un échantillon d'étudiants pragmatiques, pensant à leur futur professionnel. Cela est d'autant plus vrai que le nouveau régime est très ouvert à la coopération internationale, avec la visite remarquée du président Adama Barrow, d'abord au Sénégal, puis au Mali et enfin en France. L'offre universitaire se diversifie et de nouveaux débouchés professionnels liés aux organisations internationales, à la diplomatie, à la traduction se profilent. De ce fait, pour la majorité des étudiants interrogés, la maîtrise de la langue française devient une valeur ajoutée dans le marché du travail. Il n'est pas rare de voir nos enquêtés manifester de l'intérêt pour le français car ils veulent travailler dans la diplomatie gambienne ou encore dans les organisations internationales. La perception positive est un poids capital dans leur motivation et leur réussite dans l'apprentissage du français. Il n'en reste pas moins que l'administration universitaire, ainsi que le corps professoral gambiens devraient discuter pour pallier les difficultés évoquées. Il est vrai que ce n'est pas aux étudiants de décider du fonctionnement universitaire, mais la prise en charge de leurs représentations permettrait d’améliorer la qualité de l’enseignement. La sensibilisation par rapport à l'intérêt du français est un point essentiel pour rendre son enseignement attractif. Pour ce faire, nous soulignons la réalisation d'activités culturelles et ludiques nécessaires au renforcement des représentations positives du français. Nous avons, de plus en plus, un public estudiantin jeune dont il faut tenir compte par la proposition d'activités culturelles en adéquation et en adaptation avec leur âge : les séjours linguistiques de courtes durées sont possibles, les films courts métrages, les bandes dessinées, les jeux de mimes en classe, le karaoké, les devinettes, la chanson francophone, les reportages, la visite pédagogique en sont d'autres. 


\section{BIBLIOGRAPHIE}

Alén Garabato, C. (2011). Méthodologie de la recherche. Cours. Montpellier, Université Paul Valéry Montpellier 3.

Blanchet, Ph., Moore D., RAHAL et al. (2009). Perspectives pour une didactique, langues contextualisées. Paris : Editions des archives contemporaines.

Boyer, H. (2001). Introduction à la sociolinguistique. Paris : Editions Dunod.

Calvet, L.-J. (1999). La guerre des langues et les politiques linguistiques. Paris : Coll. Hachette.

Gomez, P. (2013). Nation et Nationalisme dans la littérature gambienne. Paris : Editions L'Harmattan.

Jobe, A. (1993). Le répertoire multilingue de l'enfant gambien : aspects sociolinguistiques et didactiques. Thèse de doctorat. Grenoble, Université de Grenoble III.

Paye, N.- M. (2012). L'émergence d'une francophonie en Gambie. Représentations, Promotion, Obstacles. Les résultats des enquêtes menées à Banjul et Brikama en 2010. Thèse de doctorat. Montpellier, Université Paul Valéry Montpellier 3.

Paye, N.- M., (1999), « La dynamique des représentations du français en Gambie », Les Cahiers de l'école doctorale en linguistique française. Brescia, Italie, 2011/5, p. 122-134.

\section{RÉSUMÉS}

Notre contribution porte sur le profil des apprenants de FLE à l'université de Gambie. Elle a pour dessein de favoriser une compréhension de ce public, de ses besoins, ses motivations, ses attentes et ses difficultés. En effet, toute orientation linguistique doit se poser sur les bases d'une enquête de terrain afin d'élucider le contexte. Pourtant dans les recherches sur la didactique du FLE en Gambie, la voix estudiantine est souvent occultée. La rédaction de cet article permettra alors d'analyser la perception estudiantine du français et de son enseignement en Gambie. Quelle est la valeur de la francophonie en Gambie selon les étudiants? Les réponses s'amorceront par une clarification de la politique universitaire en matière d'enseignement du français.

This contribution is focused in contextualized didactics. It analyzes the profile and representations of learners of French as a foreign language, at the University of The Gambia. This study shows that sociolinguistics is important and in fact, makes it possible, through the analysis of language assessments and practices, to understand motivations and to find appropriate language policies, in order to improve the conditions of teaching / learning.

\section{INDEX}

Mots-clés : français langue étrangère, politique universitaire gambienne

Thèmes : Varia

Keywords : french language, gambian university policy 


\section{AUTEUR}

\section{NDĖYE MATY PAYE}

French - Department - The University of the Gambia

Assistant-Professor à l'université de Gambie (Afrique de l'Ouest). Titulaire d'une licence en Langue et Littérature françaises et d'un master en Linguistique Française à l'université de Fès Maroc en 2005, elle obtient aussi un Master 2 en Didactique des Langues-Cultures puis un doctorat en Sciences du Langage option Sociolinguistique à l'université de Montpellier 3- France. Elle est l'auteure de quatre ouvrages et d'une dizaine d' articles.

ndeyematypaye[at]yahoo.fr 